\title{
OPEN Activating transcription factor 4 mediates adaptation of human glioblastoma cells to hypoxia and temozolomide
}

\begin{abstract}
Nadja I. Lorenz ${ }^{1,2,3,4,7}$, Alina C. M. Sittig 1,2,3,4,7, Hans Urban ${ }^{1,2,3,4}$, Anna-Luisa Luger ${ }^{1,2,3,4}$, Anna L. Engel ${ }^{1,2,3,4}$, Christian Münch ${ }^{3,5,6}$, Joachim P. Steinbach ${ }^{1,2,3,4}$ \& Michael W. Ronellenfitsch ${ }^{1,2,3,4} \bowtie$

The integrated stress response (ISR) is a central cellular adaptive program that is activated by diverse stressors including ER stress, hypoxia and nutrient deprivation to orchestrate responses via activating transcription factor 4 (ATF4). We hypothesized that ATF4 is essential for the adaptation of human glioblastoma (GB) cells to the conditions of the tumor microenvironment and is contributing to therapy resistance against chemotherapy. ATF4 induction in GB cells was modulated pharmacologically and genetically and investigated in the context of temozolomide treatment as well as glucose and oxygen deprivation. The relevance of the ISR was analyzed by cell death and metabolic measurements under conditions to approximate aspects of the GB microenvironment. ATF4 protein levels were induced by temozolomide treatment. In line, ATF4 gene suppressed GB cells (ATF4sh) displayed increased cell death and decreased survival after temozolomide treatment. Similar results were observed after treatment with the ISR inhibitor ISRIB. ATF4sh and ISRIB treated GB cells were sensitized to hypoxia-induced cell death. Our experimental study provides evidence for an important role of ATF4 for the adaptation of human GB cells to conditions of the tumor microenvironment characterized by low oxygen and nutrient availability and for the development of temozolomide resistance. Inhibiting the ISR in GB cells could therefore be a promising therapeutic approach.
\end{abstract}

With a median survival of less than one year in unselected cohorts, glioblastoma (GB) represents the most frequent, malignant primary brain tumor in adults ${ }^{1}$. So far, standard therapy is palliative and consists of surgical resection followed by alkylating chemotherapy with temozolomide and radiotherapy and optional alternating electrical fields for patients stable after radiochemotherapy ${ }^{2,3}$. For the improvement of existing therapy schemes, new approaches are urgently needed.

One reason for the poor prognosis of GB is therapy resistance-either tumor cells do not even initially respond to therapy (primary resistance) or develop resistance during the course of treatment (secondary resistance). In addition to therapeutic interventions, tumor cells are also challenged by a local decline of oxygen and nutrient supply in the GB microenvironment. Thus, the selective pressure favors tumor cells capable of mounting an effective stress response to enable adaptation and ensure cell survival. The integrated stress response (ISR) is a conserved adaptive program that can be activated by distinct stress signals including ER stress caused by the accumulation of unfolded proteins in the endoplasmic reticulum (unfolded protein response, UPR), hypoxia as well as glucose and amino acid deprivation ${ }^{4-7}$. While the ISR is primarily a pro-survival cell response, in some cellular models chronic or severe stress has also been reported to induce cell death ${ }^{5}$. Activation of the ISR is accomplished by one of four eIF2 $\alpha$ kinases (PERK, GCN2, PKR and HRI) with distinct regulatory domains and activating triggers ${ }^{7,8}$. Activation of these kinases results in phosphorylation of the eukaryotic translation initiation

\footnotetext{
${ }^{1}$ Dr. Senckenberg Institute of Neurooncology, University Hospital Frankfurt, Goethe University, Schleusenweg 2-16, 60528 Frankfurt am Main, Germany. ${ }^{2}$ German Cancer Consortium (DKTK), Partner Site Frankfurt/Mainz, Frankfurt am Main, Germany. ${ }^{3}$ Frankfurt Cancer Institute (FCI), University Hospital Frankfurt, Goethe University, Frankfurt am Main, Germany. ${ }^{4}$ University Cancer Center Frankfurt (UCT), University Hospital Frankfurt, Goethe University, Frankfurt am Main, Germany. ${ }^{5}$ Institute of Biochemistry II, Goethe University, Frankfurt am Main, Germany. ${ }^{6}$ Cardio-Pulmonary Institute, Frankfurt am Main, Germany. ${ }^{7}$ These authors contributed equally: Nadja I. Lorenz and Alina C. M. Sittig. ${ }^{凶}$ email: M.Ronellenfitsch@gmx.net
} 
factor $2 \alpha($ IF $2 \alpha)$ which in turn leads to a reduction in global translation initiation and facilitates preferential translation of selected mRNAs, including the core effector of the ISR activating transcription factor 4 (ATF4) $)^{5}$. Thus, ATF4 is frequently used as a marker for ISR activation ${ }^{5,9}$. ATF4 induction has been demonstrated in consequence of hypoxic conditions, ER stress as well as glucose and amino acid deprivation ${ }^{6,8-12}$. In GB ATF4 has not yet been studied in the context of hypoxia and combined glucose deprivation which characterize the conditions of the tumor microenvironment.

Via its leucine zipper region, ATF4 interacts with other transcription factors and activates an array of target genes including genes involved in amino acid synthesis, angiogenesis, metastasis, differentiation and drug resistance ${ }^{9}$. The termination of the stress response is regulated by a negative feedback loop that leads to dephosphorylation of eIF2a mainly by GADD $34^{9}$.

ATF4 expression has been reported to be upregulated in different types of human cancers and overexpression correlated with earlier tumor progression and therapy resistance ${ }^{13}$. Pharmacological inhibition of the ISR could be an opportunity to sensitize tumor cells to conditions of the tumor microenvironment and increase their sensitivity towards existing therapy strategies. Previous studies demonstrated that PERK inhibition itself can reduce tumor cell survival but is also associated with severe side effects in vivo especially on pancreatic function ${ }^{14}$. The small molecule inhibitor ISRIB blocks the ISR downstream of P-eIF2 $\alpha$ by targeting eIF2B, which is required as guanidine nucleotide exchange factor in the translation initiation process and thereby sustains global protein translation ${ }^{15-17}$.

Few studies on ATF4 in GB have been conducted and indicate an elevated expression in comparison to normal brain tissue ${ }^{18}$. Also in the context of chemotherapy, ATF4 has been suggested to interfere with autophagy ${ }^{19,20}$.

While induction of ATF4 by typical conditions of the GB microenvironment with severe hypoxia and low nutrient supply ${ }^{21,22}$ is plausible, the relevance of ATF4 in this context has not been investigated in detail yet ${ }^{23}$. Similarly, effects on clonal survival, the main readout to evaluate temozolomide effects in vitro have not been performed $^{18,20,24}$.

In this experimental study we investigated the regulation of ATF4, the main orchestrator of the ISR, under conditions of the glioma microenvironment. Hypoxia and temozolomide chemotherapy induced ATF4 mRNA expression and protein levels. Using pharmacological and genetic approaches we found that ATF4 directs tumor cell protective responses to mediate therapy resistance. This included protection from combined hypoxia-/ nutrient restriction-induced cell death as well as from temozolomide chemotherapy. Therefore, ATF4 could be a promising candidate for therapeutic inhibition.

\section{Results}

ATF4 is induced by pharmacological ER stress activators, glutamine depletion and temozolomide treatment in human GB cell lines. To investigate the importance of the ISR in human GB cell lines basal ATF4 mRNA expression levels were analyzed (Fig. S1a). Based on these results one GB cell line with low and one with high ATF4 mRNA expression, LNT-229 and G55 respectively, were chosen for further experiments. ATF4 mRNA levels of primary GB cells (NCH690, NCH644 and NCH421K) were comparable to the expression levels of GB cell lines. For comparison with non-tumor cells human astrocytes were investigated. Here, under basal conditions ATF4 mRNA levels were also similar to the expression levels of primary GB cells (Fig. S1a). ER stress was pharmacologically induced in LNT-229 and G55 cells with tunicamycin or thapsigargin. Treatment led to reduced cell growth after $24 \mathrm{~h}$ and even more pronounced after $96 \mathrm{~h}$ incubation (Fig. S1b). Pharmacological activation resulted in an upregulated ATF4 expression on protein level in LNT-229 and G55 cells in all tested concentrations (Fig. 1a). Additionally, both ER stress inducers led to an increased mRNA level of ATF4 itself as well as its target genes XPOT, WARS1 and TRIB3 $3^{8,25-28}$ (Fig. 1b).

ATF4 is known to be a major regulator of the ISR under amino acid deprivation conditions ${ }^{10}$. Glutamine withdrawal alone was sufficient to induce ATF4 expression in LNT-229 and G55 cells; concomitant glucose restriction did not result in an additional induction of the transcription factor (Fig. S1c, S1d). Serum withdrawal in the presence of glutamine had no effect on ATF4 expression (Fig. S1c). ATF4 itself as well as the ATF4 target genes XPOT, WARS1 and TRIB3 were transcriptionally upregulated during glutamine starvation in LNT-229 and G55 cells (Fig. S1e).

The anti-glioma chemotherapeutic temozolomide has been reported to increase ATF4 expression as a consequence of UPR induction ${ }^{18,20}$. In both, LNT-229 and G55 cells treatment with temozolomide resulted in an increased ATF4 expression compared to vehicle treated cells and a transcriptional upregulation of ATF4 and its target genes XPOT and WARS1 (Fig. 1c,d).

Gene suppression of ATF4 inhibits activation of the integrated stress response under pharmacological ER stress induction and glutamine deprivation. To investigate the effects of ATF4 in human GB cell lines LNT-229 and G55 ATF4 gene suppressed cells (ATF4sh) were generated for further analyses. Stable gene suppression was confirmed via qPCR analysis and revealed a reduction of ATF4 mRNA in both LNT-229 and G55 ATF4sh cells compared to control cells (Fig. S2a). Tunicamycin dramatically induced ATF4 expression in LNT-229 and G55 control cells (NTsh) whereas no or only a slight effect after pharmacological ER stress induction was observed in ATF4sh cells on protein (Fig. 2a) and mRNA level (Fig. 2b). Glutamine withdrawal resulted in an upregulation of ATF4 protein in LNT-229 control cells but not in the corresponding ATF4 gene-suppressed cells (Fig. S2b). This effect was also observed to a lesser extent in G55 cells most likely due to a lesser gene suppression efficiency of G55 ATF4sh cells (Fig. S2b). Glucose restriction alone did not result in an ATF4 induction under all tested conditions in both control and ATF4 gene-suppressed cells. 
a
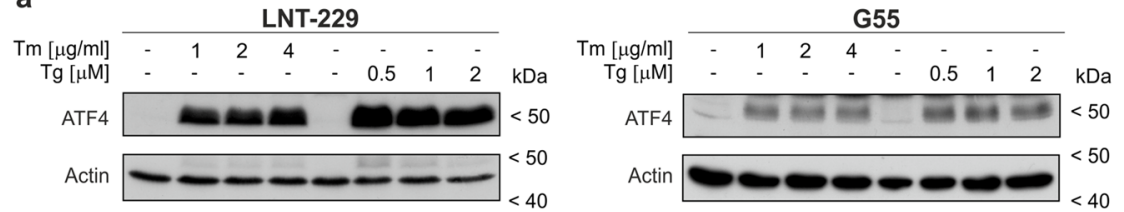

b
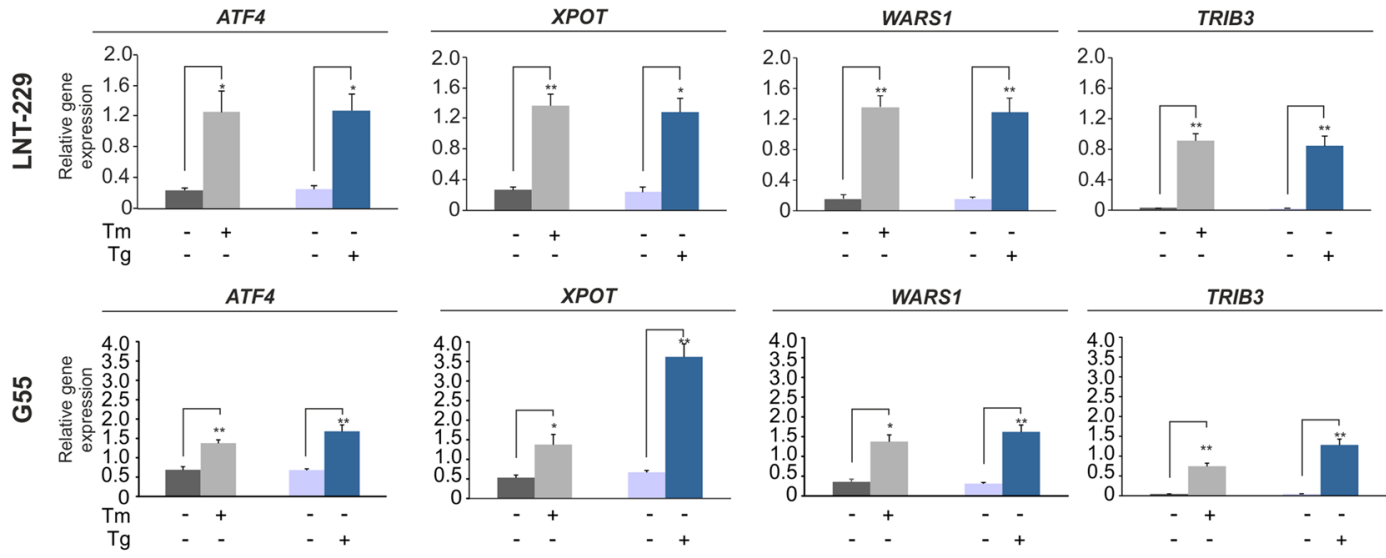

C

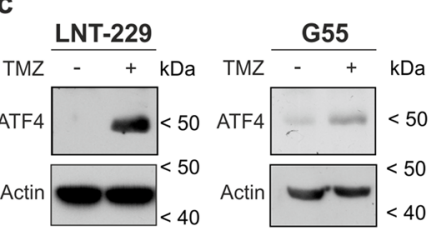

d
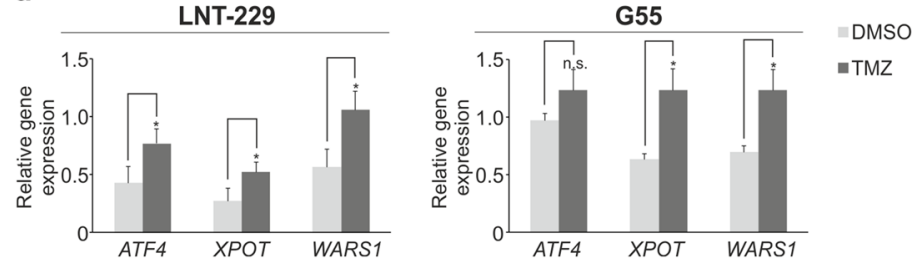

Figure 1. ATF4 is regulated by pharmacological ER stress activators and is induced by TMZ treatment in human glioblastoma cells. (a) LNT-229 and G55 cells were incubated with increasing concentration of thapsigargin (Tg) and tunicamycin (Tm) in serum-free DMEM for $8 \mathrm{~h}$. Cellular lysates were analyzed by immunoblot with antibodies for ATF4 and actin. (b) LNT-229 and G55 cells were treated with $2 \mu \mathrm{g} /$ $\mathrm{ml}$ tunicamycin $(\mathrm{Tm})$ or $1 \mu \mathrm{M}$ thapsigargin $(\mathrm{Tg})$ for $8 \mathrm{~h}$ in serum-free DMEM. cDNA was analyzed for the expression of ATF4 and its target genes XPOT, WARS 1 and TRIB3 by qPCR. $18 S$ and SDHA were used as housekeeping genes for normalization $\left(\mathrm{n}=3\right.$, mean $\pm \mathrm{SD},{ }^{\star} \mathrm{p}<0.05,{ }^{\star *} \mathrm{p}<0.01$, Student's $t$ test). (c) LNT-229 and G55 cells were treated with $400 \mu \mathrm{M}$ temozolomide (TMZ) in serum-free DMEM for $24 \mathrm{~h}$. Cellular lysates were analyzed by immunoblot with antibodies for ATF4 and actin. (d) cDNA was analyzed for induction of ATF4 and target genes XPOT and WARS1 by qPCR $\left(\mathrm{n}=3\right.$, mean $\pm \mathrm{SD}$, n.s. not significant, ${ }^{\star} \mathrm{p}<0.05$, Student's $t$ test $)$. $S D H A$ and $18 S$ were used as housekeeping genes for normalization.

ATF4 gene suppression sensitizes human GB cells to hypoxia-induced cell death and increases oxygen consumption. The GB tumor microenvironment is characterized by limited oxygen and glucose supply that drive activation of the cellular stress response machinery which enables cellular adaptation to sustain survival $^{29}$. To analyze the effects of the ER stress response in the context of hypoxia-induced cell death in GB cells we used experimental conditions mimicking the in vivo situation of reduced glucose availability and severe hypoxia $^{30}$. The relevance of ATF4 for adaptation under such conditions was investigated by pharmacological as well as genetic ATF4 modulation. Treatment with tunicamycin which induces ATF4 as shown in Fig. 1a, conferred protection towards combined hypoxia- and nutrient depletion-induced cell death in LNT-229 and G55 cells (Fig. S2c). Immunoblot analysis showed a strong induction of ATF4 only in LNT-229 control (NTsh) and only a reduced induction of ATF4 in LNT-229 ATF4sh cells after treatment under hypoxic, glucose restricted conditions (Fig. 2c). Both, LNT-229 and G55 ATF4sh cells were sensitized to hypoxia-/nutrient depletioninduced cell death compared to control cells (Fig. 2d). In line with these findings, LNT-229 and G55 ATF4sh cells displayed an increased oxygen consumption compared to NTsh cells (Fig. 2e). Conversely, treatment with the ISR inducer tunicamycin led to a reduced oxygen consumption in LNT-229 and G55 cells (Fig. S2d).

ATF4 gene suppression sensitizes GB cells to temozolomide. To investigate a potential effect of temozolomide on ATF4 expression, experiments with LNT-229 and G55 ATF4sh and corresponding NTsh cells were performed. In both cell lines, ATF4 expression was increased to a smaller extent in ATF4sh cells on protein level (Fig. 3a). In line with these results, clonogenicity assays revealed that LNT-229 and G55 ATF4sh cells displayed a reduced clonal survival confirming a higher sensitivity towards temozolomide compared to the cor- 
a

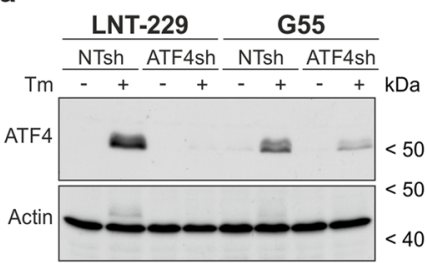

b

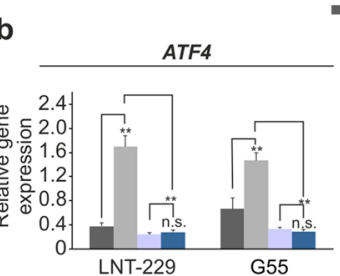

" NTsh Vehicle $=$ NTsh + Tm $=$ ATF4sh Vehicle $=$ ATF4sh + Tm
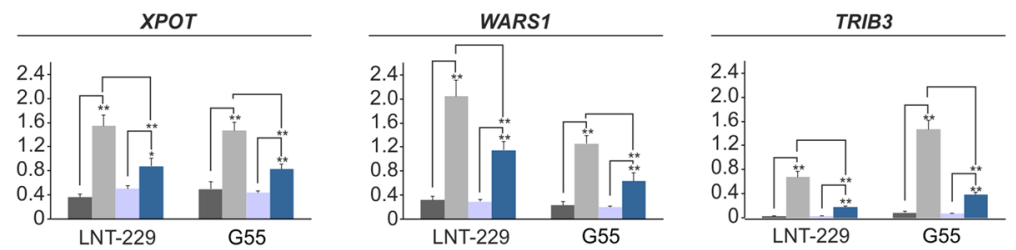

C

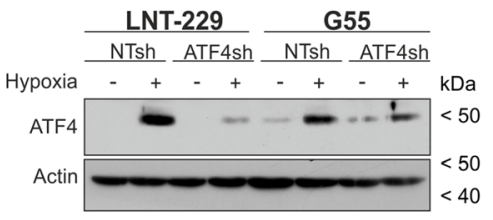

d

LNT-229

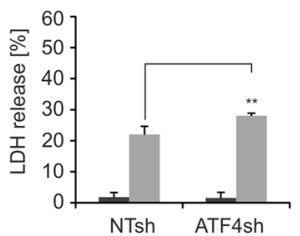

LNT-229

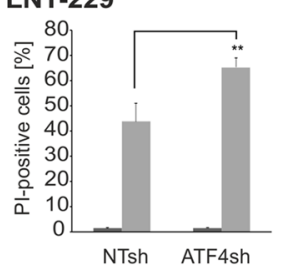

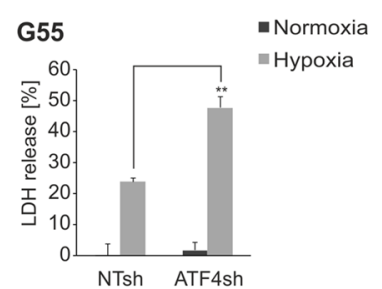

G55

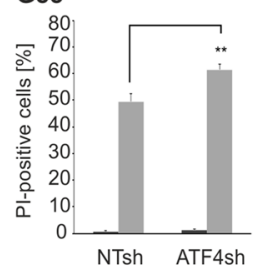

e

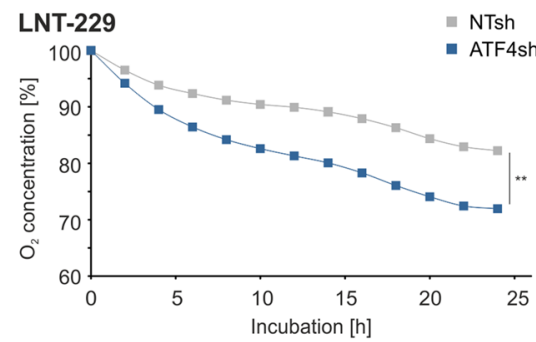

G55

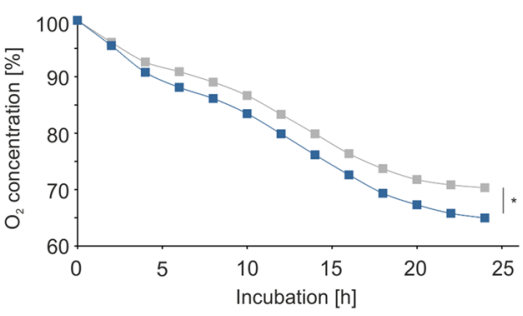

Figure 2. ATF4sh cells are insensitive for ATF4 induction via pharmacological ER stress activation and sensitized to hypoxia-induced cell death. (a) LNT-229 and G55 NTsh and ATF4sh cells were incubated with $2 \mu \mathrm{g} / \mathrm{ml}$ tunicamycin (Tm) in serum-free DMEM for $8 \mathrm{~h}$. Cellular lysates were analyzed by immunoblot with antibodies for ATF4 and actin. (b) cDNA was analyzed by qPCR with primers for ATF4, XPOT, WARS1 and TRIB3 $\left(\mathrm{n}=3\right.$, mean \pm SD, n.s. not significant, ${ }^{* *} \mathrm{p}<0.01$, Student's t-test). SDHA and $18 S$ were used as housekeeping genes for normalization. (c) LNT-229 and G55 NTsh or ATF4sh cells were incubated in serumfree DMEM containing $2 \mathrm{mM}$ glucose and $4 \mathrm{mM}$ glutamine in normoxia or hypoxia $\left(0.1 \% \mathrm{O}_{2}\right)$ for $8 \mathrm{~h}$. Cellular lysates were analyzed for the expression of ATF4 or actin by immunoblot. (d) Cells were incubated in serumfree DMEM supplemented with $2 \mathrm{mM}$ glucose and $4 \mathrm{mM}$ glutamine in normoxia or hypoxia $\left(0.1 \% \mathrm{O}_{2}\right)$. Cell death was analyzed by propidium iodide (PI) uptake and quantified by flow cytometry using BD FACS Diva software (version 6.1.3) $\left(\mathrm{n}=3\right.$, mean $\pm \mathrm{SD},{ }^{* *} \mathrm{p}<0.01$, Student's t-test) or by $\mathrm{LDH}$ release assay $(\mathrm{n}=4, \mathrm{mean} \pm \mathrm{SD}$, ${ }^{* *} \mathrm{p}<0.01$, Student's t-test). (e) Oxygen consumption of LNT-229 and G55 NTsh and ATF4sh cells was measured in serum-free DMEM supplemented with $2 \mathrm{mM}$ glucose and $4 \mathrm{mM}$ glutamine overlaid with paraffin oil with a fluorescence-based assay. Oxygen consumption is shown relative to the start of the experiment $(n=3$, mean, ${ }^{*} \mathrm{p}<0.05,{ }^{* *} \mathrm{p}<0.01$, Student's t-test). 
a

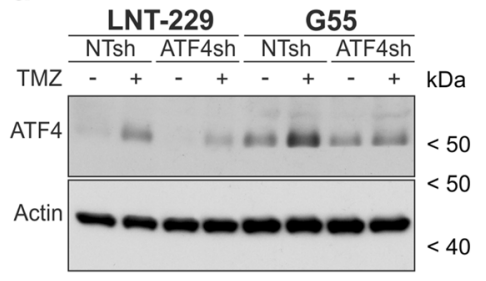

C

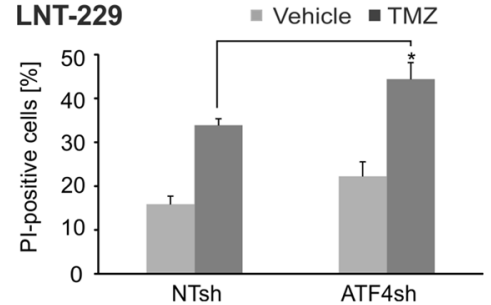

b

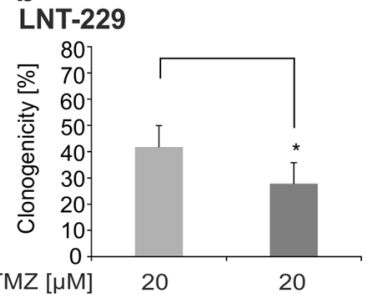

G55

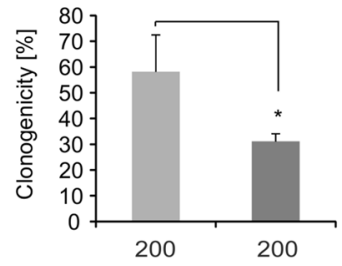

NTsh

ATF4sh

Figure 3. Gene suppression of ATF4 sensitizes human GB cells to temozolomide induced stress. (a) LNT-229 and G55 control (NTsh) or ATF4 knockdown (ATF4sh) cells were treated with $400 \mu \mathrm{M}$ temozolomide (TMZ) in serum-free DMEM for $8 \mathrm{~h}$. Cellular lysates were analyzed by immunoblot with antibodies for ATF4 and actin. (b) LNT-229 and G55 NTsh and ATF4sh cells were treated with $20 \mu \mathrm{M}$ TMZ or $200 \mu \mathrm{M}$ TMZ respectively for $24 \mathrm{~h}$ and were further allowed to grow in DMEM for 5 days. Clonogenicity is shown relative to vehicle (DMSO) $\left(\mathrm{n}=3\right.$, mean $\pm \mathrm{SD},{ }^{*} \mathrm{p}<0.05$, Student's t-test). (c) LNT-229 and G55 NTsh and ATF4sh cells were treated with $800 \mu \mathrm{M}$ TMZ for $72 \mathrm{~h}$ in serum-free DMEM. Cell death was analyzed by propidium iodide (PI) staining and quantified by flow cytometry $\left(n=3\right.$, mean $\pm S D,{ }^{*} p<0.05,{ }^{* *} p<0.01$, Student's t-test). Data were analyzed with BD FACS Diva software (version 6.1.3).

responding NTsh control (Fig. 3b). Furthermore LNT-229 ATF4sh cells showed an enhanced susceptibility to temozolomide treatment (Fig. 3c).

Pharmacological inhibition of ATF4 activation sensitizes GB cells to hypoxia induced cell death and increases temozolomide susceptibility. To further investigate the effect of ISR inhibition in the context of hypoxia induced cell death and to evaluate whether ISR inhibition could improve temozolomide treatment, we employed the pharmacological ISR inhibitor ISRIB.

While ISRIB treatment did not influence ATF4 expression in LNT-229 and G55 cells, co-treatment with tunicamycin and ISRIB resulted in decreased ATF4 expression on protein level compared to cells treated with tunicamycin alone (Fig. 4a). Similar results were observed on mRNA level of target genes where combined treatment with both substances resulted in reduced translation of ATF4 itself and ATF4 target genes XPOT and WARS1 (Fig. 4b). Under glucose restricted, hypoxic conditions, where ATF4 gene suppression resulted in increased cell death (Fig. 2d), ISR inhibition with ISRIB similarly sensitized LNT-229 and G55 cells to hypoxia induced cell death (Fig. 4c).

Temozolomide induced ATF4 activation was reduced in LNT-229 and G55 cells when ISRIB was present (Fig. 4d). ISRIB treatment did not influence cell growth in LNT-229 and G55 cells after $72 \mathrm{~h}$, but in combination with temozolomide it further decreased cell density compared to treatment with temozolomide alone (Fig. 4e). Furthermore, ISR inhibition with ISRIB significantly increased temozolomide susceptibility in LNT-229 and G55 cells after $72 \mathrm{~h}$ (Fig. 4f) in line with our results that ATF4 is important for resistance against temozolomide (Fig. 3b,c).

\section{Discussion}

Adaptation to the conditions of the tumor microenvironment is a central challenge for $\mathrm{GB}$ cells in order to enable survival and proliferation. ER stress and amino acid deprivation, frequently present in the GB microenvironment, trigger activation of the transcription factor ATF4 which induces a cellular adaptive stress response. Therefore, ATF4 is a logical candidate for therapeutic inhibition to enhance the susceptibility of GB cells towards existing therapy approaches.

Employing our paradigm of hypoxia-induced cell death with severe hypoxia and glucose restriction ${ }^{30}$, we here provide evidence that supports an important role of ATF4 and the ISR to cope with conditions found in the GB microenvironment (Fig. S3). ATF4 was induced under hypoxic conditions and pharmacological ER stress induction in human GB cells (Figs. 1a, 2c). Gene suppression of ATF4 sensitized GB cells to hypoxia induced cell death (Figs. 2c,d, S2c) indicating a protective role of ATF4 expression and induction of the ISR. In line, we found an increased oxygen consumption in ATF4sh cells indicating that these cells are more dependent on oxygen (Fig. 2e). This concept that a higher oxygen consumption correlates with enhanced sensitivity to hypoxiainduced cell death has previously been reported for PPARGC1A (PGC-1 $\alpha$ ) overexpressing GB cell ${ }^{31}$. In contrast, pharmacological ATF4 induction with tunicamycin resulted in a reduced oxygen consumption compared to 
a

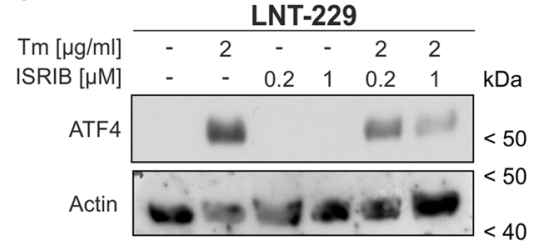

G55

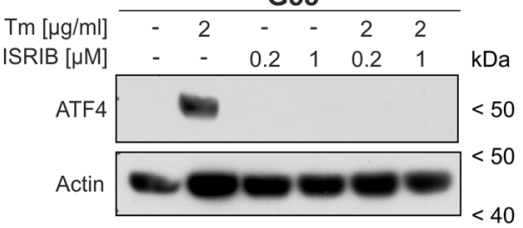

b
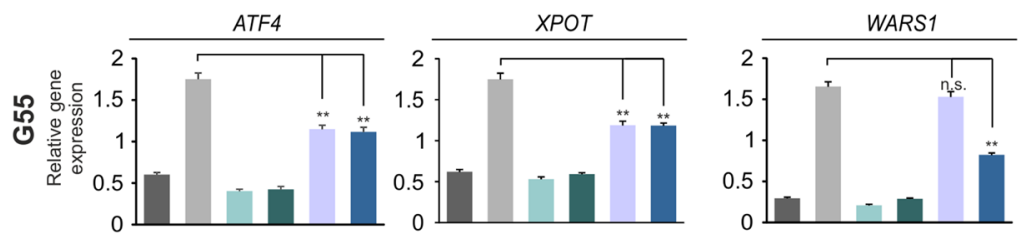

- Vehicle

- $\mathrm{Tm}$

= $0.2 \mu \mathrm{M}$ ISRIB

- $1 \mu \mathrm{M}$ ISRIB

$=\mathrm{Tm}+0.2 \mu \mathrm{M}$ ISRIB

- Tm + $1 \mu \mathrm{M}$ ISRIB

C

LNT-229

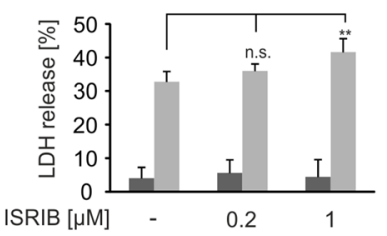

G55

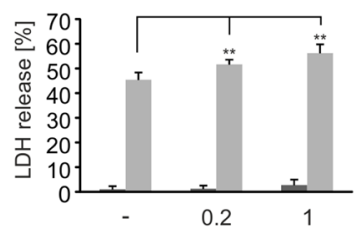

- Normoxia

-Hypoxia

\section{d}
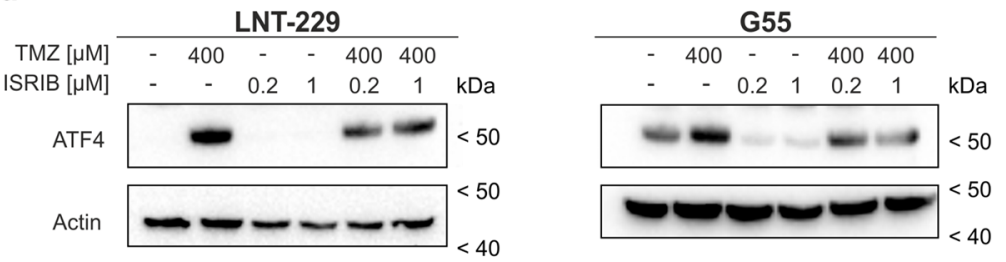

e

LNT-229

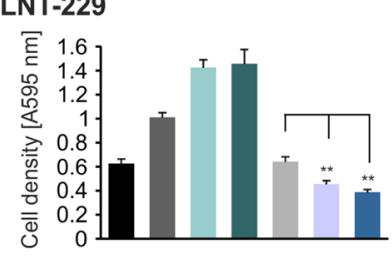

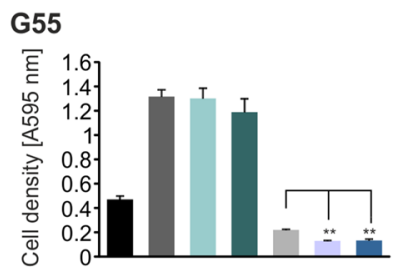

- T0

- Vehicle

$\because 0.2 \mu \mathrm{M}$ ISRIB

- $1 \mu \mathrm{M}$ ISRIB

- $400 \mu \mathrm{M}$ TMZ

$=400 \mu \mathrm{M}$ TMZ + $0.2 \mu \mathrm{M}$ ISRIB

- $400 \mu \mathrm{M} \mathrm{TMZ}+1 \mu \mathrm{M}$ ISRIB

f

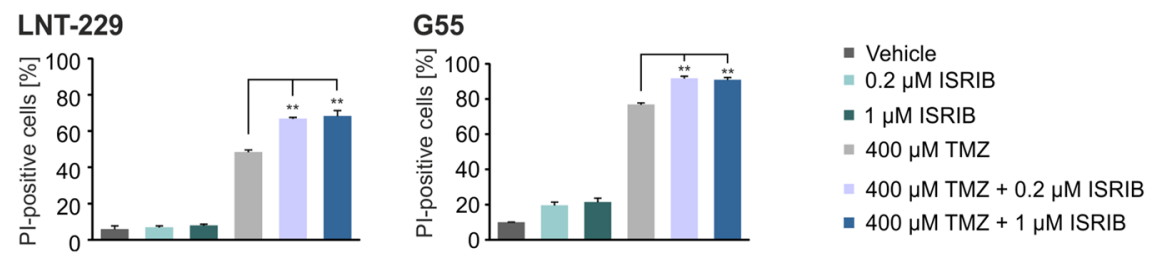

Figure 4. ISRIB treatment sensitizes human GB cells to hypoxia induced cell death and increases temozolomide toxicity. (a) LNT-229 and G55 cells were treated with $2 \mu \mathrm{g} / \mathrm{ml}$ tunicamycin (Tm), $0.2 \mu \mathrm{M}$ or $1 \mu \mathrm{M}$ ISRIB or a combination of both substances in serum-free DMEM for $8 \mathrm{~h}$ as indicated. Cellular lysates were analyzed by immunoblot with antibodies for ATF4 and actin. (b) Isolated cDNA of G55 cells was analyzed by qPCR for the expression of ATF4, XPOT and WARS1. SDHA and $18 S$ were used for normalization $\left(\mathrm{n}=3\right.$, mean \pm SD, n.s. not significant, ${ }^{* *} \mathrm{p}<0.01$, Student's t-test). (c) LNT-229 and G55 cells were incubated in serum-free DMEM without glutamine in normoxia or hypoxia $\left(0.1 \% \mathrm{O}_{2}\right)$. Cell death was analyzed by $\mathrm{LDH}$ release assay $\left(\mathrm{n}=4\right.$, mean \pm SD, n.s. not significant, ${ }^{* *} \mathrm{p}<0.01$, Student's t-test). (d) Cellular lysates of LNT-229 and G55 cells treated with temozolomide and ISRIB for $24 \mathrm{~h}$ in serum-free DMEM as indicated were analyzed by immunoblot with antibodies for ATF4 and actin. (e) LNT-229 and G55 cells were treated for $72 \mathrm{~h}$ in serum-free DMEM as indicated. Cell densities were measured by CV staining $\left(n=3\right.$, mean $\pm S D$, ${ }^{* *} \mathrm{p}<0.01$, Student's t-test). (f) Cell death of LNT-229 and G55 cells was analyzed by propidium iodide (PI) uptake and quantified by flow cytometry $\left(\mathrm{n}=3\right.$, mean $\pm \mathrm{SD},{ }^{*} \mathrm{p}<0.01$, Student's $\mathrm{t}$-test) after treatment with temozolomide and ISRIB in serum-free DMEM for $72 \mathrm{~h}$ as indicated. Data analysis was performed with BD FACS Diva software (version 6.1.3). 
vehicle treated LNT-229 and G55 cells (Fig. S2d) indicating that GB cells with an intact ISR reduce their oxygen consumption during ER stress conditions. Interestingly, it has recently been reported that the stress responses mediated by the ISR and via mTOR display a significant overlap in their translational programs ${ }^{32}$. In line with this finding, we have previously shown that a defective mTOR inhibition sensitizes GB cells to hypoxia-induced cell death ${ }^{33}$ in a similar manner as a defective ISR (Fig. 2d). In parallel, dysfunction of both stress responses was associated with an increased oxygen consumption (Fig. 2e $)^{33}$.

Pharmacological ER stress induction with tunicamycin and thapsigargin is known to induce ATF4 and a number of ATF4 target genes ${ }^{8,25-28}$. In our experiments, treatment with tunicamycin as well as thapsigargin led to a significant increase of ATF4 and its target genes in human GB cell lines (Fig. 1a,b). This effect was strongly reduced in ATF4sh cells compared to control cells in LNT-229 and G55 (Fig. 2a,b). Furthermore, we could demonstrate that ATF4 was induced by glutamine depletion in GB cells whereas glucose starvation alone did not modulate ATF4 expression (Fig. S1c,d). This finding is consistent with data showing an upregulation of ATF4 via the GCN2/P-eIF2 $\alpha / A T F 4$ axis during glutamine starvation conditions in colorectal adenocarcinoma and fibrosarcoma cell lines ${ }^{10}$. Glutamine is an important non-essential amino acid used by cancer cells not only for the synthesis of nucleotides and proteins but also as an energy source for ATP production and to support glutathione biosynthesis in redox stress conditions ${ }^{34}$. It has been reported that glutamine depletion led to a $50 \%$ reduction in survival in ATF4sh cells compared to a $25 \%$ reduction in control cells ${ }^{10}$. Our results emphasize the role of glutamine depletion in the context of the ISR in human GB cells where an ATF4 upregulation possibly helps the tumor cells to adjust to altered nutrient availability.

Previous studies in human GB cells have shown that ATF4 expression is induced by the alkylating chemotherapeutic agent temozolomide ${ }^{18,20}$. In our experiments we found an ATF4 dependent ISR activation after temozolomide treatment in human GB cell lines (Fig. 1c,d) which was strongly reduced in ATF4sh cells (Fig. 3a). Furthermore, gene suppression of ATF4 led to a reduction of clonogenicity after temozolomide treatment in LNT-229 and G55 cells compared to the corresponding control cells (Fig. 3b). The fact that the impact of temozolomide treatment on the clonogenicity is more pronounced in G55 cells could be due to the higher basal level of ATF4 (Fig. S1a) and therefore a higher dependency of those cells on ATF4 activity. ATF4sh cells were also more susceptible to temozolomide induced acute cell death compared to control cells (Fig. 3c). Thus, our results demonstrate that initiation of the ISR and induction of ATF4 are essential for the activation of cellular resistance mechanisms which allow GB cells to overcome temozolomide toxicity (Fig. S3). In previous studies it has been demonstrated that PERK inhibition can be beneficial for cancer treatment ${ }^{14}$. While many ISR inhibitors target PERK itself, ISRIB specifically inhibits the stress response downstream of PERK by reversing the attenuation of eIF2B by phosphorylated eIF2 $\alpha^{15-17}$. PERK inhibition was reported to lead to side effects in in vivo models, especially on pancreatic function, whereas targeting phosphorylated eIF2a with ISRIB does not show pancreatic toxicity ${ }^{14,35}$. ISRIB treatment sensitized GB cells to hypoxia induced cell death (Fig. 4c), indicating that ISR inhibition leads to an increased vulnerability to conditions of the tumor microenvironment (hypoxia, nutrient deprivation) and impaired tumor cell survival. Furthermore, our experiments using combined temozolomide and ISRIB treatment highlight the importance of the ISR and especially ATF4 induction for temozolomide resistance in GB cells. ISR inhibition enhanced temozolomide susceptibility in GB cell lines (Fig. 4e,f).

While the ISR inducing agents thapsigargin and tunicamycin are frequently used to study ATF4-mediated cellular effects, these substances are certainly not specific for ATF4. Also with regard to shRNA approaches off-target effects cannot be ruled out. Nevertheless, our work provides consistent evidence that supports an important role of eIF2 $\alpha$-ATF4 axis and the integrated stress response of GB cells to conditions found in the tumor microenvironment (low glucose and hypoxia) and in response to temozolomide.

Analyses of databases suggested elevated ATF4 expression in gliomas in comparison to normal brain tissue and an association of increased ATF4 transcription with poor overall survival in glioma cohorts ${ }^{18,36}$. Several in vitro investigations have implicated ATF4 in tumor cell survival with sometimes opposing cytoprotective and cytotoxic roles depending on context: ATF4 has been shown to attenuate ferroptosis under dihydroartemisinininduced ER stress or sorafenib treatment to sustain glioma cell survival and inhibition of this axis was therefore proposed as a therapeutic strategy ${ }^{37}$. On the contrary, another study in GB cells demonstrated that ATF4 played a key role to induce autophagic cell death via reticulophagy (i.e. the selective degradation of the endoplasmic reticulum) in response to loperamide treatment and in this context activation of ATF4 was suggested to be beneficial for tumor therapy ${ }^{38}$. These studies highlight the ambiguous character of the ATF4 mediated stress response depending on the experimental context. A more in depth understanding of ATF4 effects is therefore urgently needed before advancing ATF4 targeting strategies to clinical trials. In our study we approximated in vivo conditions both with regard to metabolic (oxygen and glucose deprivation) and treatment (with temozolomide as the standard chemotherapeutic for gliomas) scenarios. In both scenarios ATF4 mediated protective adaptation in GB cells. A next step is to validate the advantages of a combined temozolomide and anti-ATF4 (e.g. ISRIB treatment) approach in in vivo GB mouse models.

Our preclinical results have potentially high translational value. Primary or secondary resistance to temozolomide is a major problem in GB treatment. Several scenarios of combining temozolomide with an ISR inhibitor (e.g. ISRIB) could be of interest: including ISR inhibition directly in the first line temozolomide treatment algorithm could enhance temozolomide efficacy particularly in MGMT-promoter methylated tumors and maybe also help achieve some efficacy in MGMT unmethylated tumors. Another scenario could be to include ISR inhibition as a treatment concept to (re-)sensitize GB to temozolomide in the recurrent disease setting. In this context, it would be interesting to analyze histology specimens of recurrent gliomas after temozolomide treatment for activation of the ISR which would be a rationale for such an approach in the recurrent disease setting.

The ISR has gained interest across the cancer field and it is likely that candidates for clinical testing will become available. Therapeutic inhibition could be a promising clinical strategy to improve treatment of GBs. 


\begin{tabular}{|l|l|l|l|l|}
\hline & MGMT & IDH & p53 & PTEN \\
\hline LNT-229 & Methylated & Wildtype & Wildtype & Wildtype \\
\hline G55 & Unmethylated & Wildtype & Mutant & n.a \\
\hline
\end{tabular}

Table 1. Genetic background of LNT-229 and G55 cells. MGMT, IDH, p53 and PTEN status of G55 and LNT229 cells $^{33}$.

\section{Material and methods}

Reagents, cell lines and culture conditions. Tunicamycin and thapsigargin were purchased from Tocris (Bristol, UK). All reagents not specified elsewhere were purchased from Sigma (Taufkirchen, Germany). LNT-229 cells were a kind gift of N. de Tribolet (Lausanne, Switzerland). LNT-229 cells were authenticated using STR analysis by Multiplexion (Heidelberg, Germany). The STR profile of the tested cells matched with the known profile for LN-229. LNT-229 and LN-229 cells only differ in their p53 status ${ }^{39}$. G55 T2 cells (named G55 for simplicity throughout the manuscript) were a kind gift of Manfred Westphal and Kathrin Lamszus (Hamburg, Germany $)^{40}$, a STR profile for this cell line has not been deposited in databases yet. MGMT, IDH, p53 and PTEN status of LNT-229 and G55 cells have been described previously and are listed (Table 1) 33,41 .

All cell lines were regularly checked for mycoplasma contamination throughout the study and only contamination-free cells were used for experiments.

Cell lines were cultured in Dulbecco's modified Eagle's medium (DMEM) containing 10\% fetal calf serum (Biochrom KG, Berlin, Germany), $100 \mathrm{IU} / \mathrm{ml}$ penicillin and $100 \mu \mathrm{g} / \mathrm{ml}$ streptomycin (Life Technologies, Karlsruhe, Germany) in cell culture incubators at $37^{\circ} \mathrm{C}$ and $5 \% \mathrm{CO}_{2}^{42}$. For experiments comparing sub-cell lines, equal cell densities at the start of the experiment were confirmed by crystal violet (CV) staining in parallel assays $^{42}$.

Human primary GB cells NCH690, NCH421K and NCH644 were purchased from CLS (Eppelheim, Germany) and were cultured in Neurobasal A medium (Thermo Fisher Scientific, Dreieich, Germany) supplemented with $1 \times$ B27 supplement (Thermo Fisher Scientific, Dreieich, Germany), 2 mM glutamine (Thermo Fisher Scientific, Dreieich, Germany), $1 \mathrm{U} / \mathrm{ml}$ heparin, $100 \mathrm{IU} / \mathrm{ml}$ penicillin and $100 \mu \mathrm{g} / \mathrm{ml}$ streptomycin (Life Technologies, Karlsruhe, Germany) and $20 \mathrm{ng} / \mathrm{ml}$ EGF and FGF-2 (ReliaTech GmbH, Wolfenbüttel, Germany). Human astrocytes were purchased from Innoprot (Derio, Spain) and were cultured in Astrocyte medium (Innoprot, Derio, Spain) according to the manufacturer's protocol.

Temozolomide treatment. The different temozolomide concentrations were chosen based on the experimental setup and cell line characteristics (i.e. MGMT promoter methylation status). The effect of low doses of temozolomide on clonal survival are a known phenomenon and are usually by several factors lower than doses required to induce acute toxicity ${ }^{43,44}$. For clonal survival analyses of MGMT promoter methylated LNT-229 cells $20 \mu \mathrm{M}$ temozolomide and for MGMT promoter unmethylated G55 cells $200 \mu \mathrm{M}$ temozolomide were chosen. For acute toxicity cells were treated with higher doses of temozolomide for $72 \mathrm{~h}$. To investigate acute effects of temozolomide treatment on mRNA expression and protein levels, cells were incubated with $400 \mu \mathrm{M}$ temozolomide for $8 \mathrm{~h}$.

Generation of ATF4 gene suppressed cells. pLKO.1 plasmids targeting ATF4 (ATF4sh, TRCN0000329695) and non-targeting control (NTsh, \#1864) were purchased from Sigma and Addgene respectively. HEK293 cells were used for lentiviral production with pCMV-dR8.2 dvpr (Addgene, \#8455) and pCMVVSVG (Addgene, \#8454) as packaging and envelope plasmids according to the manufacturer's protocol. Lentiviral transduction was performed using polybrene (Merck Millipore, Darmstadt, Germany). ATF4sh and NTsh cells were cultured in standard medium supplemented with $2 \mu \mathrm{g} / \mathrm{ml}$ puromycin (AppliChem, Darmstadt, Germany). Experiments were performed with early passage pooled clones.

Induction of hypoxia. Cells were seeded and allow to attach overnight under normoxia. Experiments were performed in serum-free DMEM containing $2 \mathrm{mM}$ glucose and $4 \mathrm{mM}$ glutamine under normoxia or $0.1 \% \mathrm{O}_{2}$ hypoxia in BD GasPak pouches (Becton-Dickinson, Heidelberg, Germany) for anaerobic culture for the indicated intervals ${ }^{33,45}$.

Immunoblot. Cell lysate preparation and immunoblot analysis was performed as described previously ${ }^{33}$. Primary antibodies for ATF4 (Proteintech, Rosemont, IL, USA, 10835-1-AP and Cell Signaling Technology, Danvers, MA, USA, \#11815) or actin (Santa Cruz Biotechnology, Santa Cruz, CA, USA, \#sc-1616) were employed as indicated. Secondary anti-rabbit and anti-goat antibodies were purchased from Jackson ImmunoResearch (West Grove, PA, USA, \#111-036-144) and Santa Cruz (\#sc-2020). Chemiluminescence was used for detection. All full length immunoblots are shown in Fig. S4.

RNA extraction and quantitative PCR analysis. Total RNA was extracted using TRIzol and EXTRACTME RNA isolation kit (Blirt, Gdansk, Poland). cDNA was synthesized using the Vilo cDNA synthesis kit (Invitogen, Carlsbad, CA, USA) ${ }^{33}$. Quantitative RT-PCR (qPCR) was performed using the Absolute Blue SYBR Green Fluorescein q-PCR Mastermix (Thermo Fisher Scientific, Dreieich, Germany) with the correspond- 
ing primer pairs (Table S1). $18 S$ and SDHA were used as housekeeping genes for normalization. Data was analyzed using the Vandesompele method ${ }^{46}$.

Cell density and viability assays. For cell growth analyses cells were seeded in 96-well plates and allowed to attach overnight. After treatment, cell density was analyzed by CV staining after the indicated intervals as previously described ${ }^{42,47}$. Cell viability was analyzed by lactate dehydrogenase release assays with the Cytotoxicity Detection Kit (LDH) (Roche, Mannheim, Germany) or by propidium iodide (PI) uptake assays via flow cytometry as previously described ${ }^{30,33}$. Data were acquired with a BD Canto II flow cytometer and analyzed with BD FACS Diva software (version 6.1.3).

Clonal survival assays. For analysis of clonal survival 500 cells were seeded per well of a 6 well plate and allowed to attach overnight. Cells were treated with temozolomide in serum-free DMEM. Medium was replaced with fresh DMEM containing 10\% FCS after $24 \mathrm{~h}$. CV staining was performed 5 days later. Clones were counted manually ${ }^{48}$.

Oxygen consumption. Cells were seeded in 24-well plates with oxygen sensors (PreSens, Regensburg, Germany) and allowed to attach overnight. Medium was replaced and cells were overlaid with sterile paraffin oil. Oxygen consumption was measured in optical sensor plates and concentrations of remaining oxygen were recorded over time ${ }^{33}$. For statistical analysis values of the end-points of the experiments were analyzed by a twotailed student's t-tests.

Statistics. Quantitative data are expressed as mean and standard deviation (SD). A two-tailed student's t-test was used for the calculation of $\mathrm{p}$-values. Values of $\mathrm{p}<0.01$ were considered as highly significant, $\mathrm{p}<0.05$ as significant and $\mathrm{p}>0.05$ as not significant (n.s.).

\section{Data availability}

The datasets used or analyzed during the current study are available from the corresponding author on reasonable request.

Received: 18 February 2021; Accepted: 18 June 2021

Published online: 08 July 2021

\section{References}

1. Ohgaki, H. et al. Genetic pathways to glioblastoma: a population-based study. Can. Res. 64, 6892-6899. https://doi.org/10.1158/ 0008-5472.CAN-04-1337 (2004).

2. Stupp, R. et al. Radiotherapy plus concomitant and adjuvant temozolomide for glioblastoma. N. Engl. J. Med. 352, 987-996. https:// doi.org/10.1056/NEJMoa043330 (2005).

3. Stupp, R. et al. Effect of tumor-treating fields plus maintenance temozolomide vs maintenance temozolomide alone on survival in patients with glioblastoma: A randomized clinical trial. JAMA 318, 2306-2316. https://doi.org/10.1001/jama.2017.18718 (2017).

4. PeñarandaFajardo, N. M., Meijer, C. \& Kruyt, F. A. E. The endoplasmic reticulum stress/unfolded protein response in gliomagenesis, tumor progression and as a therapeutic target in glioblastoma. Biochem. Pharmacol. 118, 1-8. https://doi.org/10.1016/j.bcp.2016. 04.008 (2016)

5. Pakos-Zebrucka, K. et al. The integrated stress response. EMBO Rep. 17, 1374-1395. https://doi.org/10.15252/embr.201642195 (2016).

6. Rzymski, T. et al. Regulation of autophagy by ATF4 in response to severe hypoxia. Oncogene 29, 4424-4435. https://doi.org/10. 1038/onc.2010.191(2010).

7. Wek, R. C., Jiang, H.-Y. \& Anthony, T. G. Coping with stress: eIF2 kinases and translational control. Biochem. Soc. Trans. 34, 7-11. https://doi.org/10.1042/BST20060007 (2006).

8. Harding, H. P. et al. Regulated translation initiation controls stress-induced gene expression in mammalian cells. Mol. Cell 6, 1099-1108. https://doi.org/10.1016/S1097-2765(00)00108-8 (2000).

9. Ameri, K. \& Harris, A. L. Activating transcription factor 4. Int. J. Biochem. Cell Biol. 40, 14-21. https://doi.org/10.1016/j.biocel. 2007.01.020 (2008).

10. Ye, J. et al. The GCN2-ATF4 pathway is critical for tumour cell survival and proliferation in response to nutrient deprivation. EMBO J. 29, 2082-2096. https://doi.org/10.1038/emboj.2010.81 (2010).

11. Blais, J. D. et al. Activating transcription factor 4 is translationally regulated by hypoxic stress. Mol. Cell. Biol. 24, 7469-7482. https://doi.org/10.1128/MCB.24.17.7469-7482.2004 (2004).

12. Iurlaro, R. et al. Glucose deprivation induces ATF4-mediated apoptosis through TRAIL death receptors. Mol. Cell. Biol. https:// doi.org/10.1128/MCB.00479-16 (2017).

13. Singleton, D. C. \& Harris, A. L. Targeting the ATF4 pathway in cancer therapy. Expert Opin. Ther. Targets 16, 1189-1202. https:// doi.org/10.1517/14728222.2012.728207 (2012)

14. Atkins, C. et al. Characterization of a novel PERK kinase inhibitor with antitumor and antiangiogenic activity. Can. Res. 73, 1993-2002. https://doi.org/10.1158/0008-5472.CAN-12-3109 (2013).

15. Rabouw, H. H. et al. Small molecule ISRIB suppresses the integrated stress response within a defined window of activation. Proc. Natl. Acad. Sci. USA. 116, 2097-2102. https://doi.org/10.1073/pnas.1815767116 (2019).

16. Sidrauski, C. et al. Pharmacological brake-release of mRNA translation enhances cognitive memory. Elife https://doi.org/10.7554/ eLife.00498 (2013).

17. Sidrauski, C., McGeachy, A. M., Ingolia, N. T. \& Walter, P. The small molecule ISRIB reverses the effects of eIF2 $\alpha$ phosphorylation on translation and stress granule assembly. Elife https://doi.org/10.7554/eLife.05033 (2015).

18. Chen, D. et al. ATF4 promotes angiogenesis and neuronal cell death and confers ferroptosis in a xCT-dependent manner. Oncogene 36, 5593-5608. https://doi.org/10.1038/onc.2017.146 (2017).

19. He, Y. et al. Regulation of integrated stress response sensitizes U87MG glioblastoma cells to temozolomide through the mitochondrial apoptosis pathway. Anat. Rec. 301, 1390-1397. https://doi.org/10.1002/ar.23839 (2018).

20. Chen, D., Rauh, M., Buchfelder, M., Eyupoglu, I. Y. \& Savaskan, N. The oxido-metabolic driver ATF4 enhances temozolamide chemo-resistance in human gliomas. Oncotarget 8, 51164-51176. https://doi.org/10.18632/oncotarget.17737 (2017). 
21. Evans, S. M. et al. Hypoxia is important in the biology and aggression of human glial brain tumors. Clin. Cancer Res. 10, 8177-8184. https://doi.org/10.1158/1078-0432.CCR-04-1081 (2004).

22. Monteiro, A. R., Hill, R., Pilkington, G. J. \& Madureira, P. A. The role of hypoxia in glioblastoma invasion. Cells https://doi.org/ $10.3390 /$ cells6040045 (2017).

23. Moeckel, S. et al. ATF4 contributes to autophagy and survival in sunitinib treated brain tumor initiating cells (BTICs). Oncotarget 10, 368-382. https://doi.org/10.18632/oncotarget.26569 (2019).

24. Weatherbee, J. L., Kraus, J.-L. \& Ross, A. H. ER stress in temozolomide-treated glioblastomas interferes with DNA repair and induces apoptosis. Oncotarget 7, 43820-43834. https://doi.org/10.18632/oncotarget.9907 (2016).

25. Han, J. et al. ER-stress-induced transcriptional regulation increases protein synthesis leading to cell death. Nat. Cell Biol. 15, 481-490. https://doi.org/10.1038/ncb2738 (2013).

26. Ohoka, N., Yoshii, S., Hattori, T., Onozaki, K. \& Hayashi, H. TRB3, a novel ER stress-inducible gene, is induced via ATF4-CHOP pathway and is involved in cell death. EMBO J. 24, 1243-1255. https://doi.org/10.1038/sj.emboj.7600596 (2005).

27. Armstrong, J. L., Flockhart, R., Veal, G. J., Lovat, P. E. \& Redfern, C. P. F. Regulation of endoplasmic reticulum stress-induced cell death by ATF4 in neuroectodermal tumor cells. J. Biol. Chem. 285, 6091-6100. https://doi.org/10.1074/jbc.M109.014092 (2010).

28. Fusakio, M. E. et al. Transcription factor ATF4 directs basal and stress-induced gene expression in the unfolded protein response and cholesterol metabolism in the liver. Mol. Biol. Cell 27, 1536-1551. https://doi.org/10.1091/mbc.E16-01-0039 (2016).

29. Jawhari, S., Ratinaud, M.-H. \& Verdier, M. Glioblastoma, hypoxia and autophagy: A survival-prone "ménage-à-trois". Cell Death Dis. 7, e2434. https://doi.org/10.1038/cddis.2016.318 (2016).

30. Steinbach, J. P., Wolburg, H., Klumpp, A., Probst, H. \& Weller, M. Hypoxia-induced cell death in human malignant glioma cells: energy deprivation promotes decoupling of mitochondrial cytochrome $c$ release from caspase processing and necrotic cell death. Cell Death Differ. 10, 823-832. https://doi.org/10.1038/sj.cdd.4401252 (2003).

31. Bruns, I. et al. Disruption of peroxisome proliferator-activated receptor $\gamma$ coactivator (PGC)-1a reverts key features of the neoplastic phenotype of glioma cells. J. Biol. Chem. 294, 3037-3050. https://doi.org/10.1074/jbc.RA118.006993 (2019).

32. Klann, K., Tascher, G. \& Münch, C. Functional translatome proteomics reveal converging and dose-dependent regulation by mTORC1 and eIF2a. Mol. Cell 77, 913-925.e4. https://doi.org/10.1016/j.molcel.2019.11.010 (2020).

33. Thiepold, A.-L. et al. Mammalian target of rapamycin complex 1 activation sensitizes human glioma cells to hypoxia-induced cell death. Brain 140, 2623-2638. https://doi.org/10.1093/brain/awx196 (2017).

34. Jiang, J., Srivastava, S. \& Zhang, J. Starve cancer cells of glutamine: Break the spell or make a hungry monster?. Cancers https:// doi.org/10.3390/cancers11060804 (2019).

35. Halliday, M. et al. Partial restoration of protein synthesis rates by the small molecule ISRIB prevents neurodegeneration without pancreatic toxicity. Cell Death Dis. 6, e1672. https://doi.org/10.1038/cddis.2015.49 (2015).

36. Peñaranda-Fajardo, N. M. et al. ER stress and UPR activation in glioblastoma: identification of a noncanonical PERK mechanism regulating GBM stem cells through SOX2 modulation. Cell Death Dis. 10, 690. https://doi.org/10.1038/s41419-019-1934-1 (2019).

37. Chen, Y. et al. Dihydroartemisinin-induced unfolded protein response feedback attenuates ferroptosis via PERK/ATF4/HSPA5 pathway in glioma cells. J. Exp. Clin. Cancer Res. 38, 402. https://doi.org/10.1186/s13046-019-1413-7 (2019).

38. Zielke, S. et al. ATF4 links ER stress with reticulophagy in glioblastoma cells. Autophagy 1, 1-17. https://doi.org/10.1080/15548 $627.2020 .1827780(2020)$.

39. Wischhusen, J., Naumann, U., Ohgaki, H., Rastinejad, F. \& Weller, M. CP-31398, a novel p53-stabilizing agent, induces p53-dependent and p53-independent glioma cell death. Oncogene 22, 8233-8245. https://doi.org/10.1038/sj.onc.1207198 (2003).

40. Eckerich, C. et al. RON receptor tyrosine kinase in human gliomas: Expression, function, and identification of a novel soluble splice variant. J. Neurochem. 109, 969-980. https://doi.org/10.1111/j.1471-4159.2009.06027.x (2009).

41. Ishii, N. et al. Frequent co-alterations of TP53, p16/CDKN2A, p14ARF, PTEN tumor suppressor genes in human glioma cell lines. Brain Pathol. 9, 469-479. https://doi.org/10.1111/j.1750-3639.1999.tb00536.x (1999).

42. Ronellenfitsch, M. W. et al. Antagonism of the mammalian target of rapamycin selectively mediates metabolic effects of epidermal growth factor receptor inhibition and protects human malignant glioma cells from hypoxia-induced cell death. Brain 132, 1509-1522. https://doi.org/10.1093/brain/awp093 (2009).

43. Hermisson, M. et al. O6-methylguanine DNA methyltransferase and $\mathrm{p} 53$ status predict temozolomide sensitivity in human malignant glioma cells. J. Neurochem. 96, 766-776. https://doi.org/10.1111/j.1471-4159.2005.03583.x (2006).

44. Gramatzki, D. et al. Glioma cell death induced by irradiation or alkylating agent chemotherapy is independent of the intrinsic ceramide pathway. PLoS ONE 8, e63527. https://doi.org/10.1371/journal.pone.0063527 (2013).

45. Wanka, C. et al. Synthesis of cytochrome C oxidase 2: A p53-dependent metabolic regulator that promotes respiratory function and protects glioma and colon cancer cells from hypoxia-induced cell death. Oncogene 31, 3764-3776. https://doi.org/10.1038/ onc.2011.530 (2012).

46. Vandesompele, J. et al. Accurate normalization of real-time quantitative RT-PCR data by geometric averaging of multiple internal control genes. Genome Biol. 3, 0034. https://doi.org/10.1186/gb-2002-3-7-research0034 (2002).

47. Grady, J. E., Lummis, W. L. \& Smith, C. G. An improved tissue culture assay. III. Alternate methods for measuring cell growth. Cancer Res. 20, 1114-1117 (1960).

48. Fischer, S. et al. Hypoxia enhances the antiglioma cytotoxicity of B10, a glycosylated derivative of betulinic acid. PLoS ONE $\mathbf{9}$, e94921. https://doi.org/10.1371/journal.pone.0094921 (2014).

\section{Author contributions}

M.W.R. and J.P.S. conceived the study and designed the experiments. A.C.M.S., N.I.L., H.U., A.-L.L., A.L.E. and M.W.R. performed the experiments. A.C.M.S., N.I.L., H.U., A.-L.L., A.L.E., C.M. and M.W.R. analyzed the data. A.C.M.S., N.I.L. and M.W.R. wrote the manuscript. All authors helped to draft the manuscript and read and approved the final version.

\section{Funding}

Open Access funding enabled and organized by Projekt DEAL. The Senckenberg Institute of Neurooncology is supported by the Senckenberg Foundation. A.-L.L. has received funding from the Frankfurt Research Funding (FFF) of the Medical Faculty of the Goethe University Frankfurt (program "Patenschaftsmodell") and a "Clinician Scientist” fellowship from the Else Kröner-Forschungskolleg (EKF). C.M. acknowledges funding from the German Research Foundation (DFG) Emmy Noether Program (MU 4216/1-1). J.P.S. and M.W.R. received funding by the State of Hessen within the LOEWE program. M.W.R also received funding from the Frankfurt Research Funding (FFF) (programs "Nachwuchsforscher" \& "Clinician Scientists") as well as a fellowship by the University Cancer Center Frankfurt (UCT). 


\section{Competing interests}

JPS has a consulting or advisory board membership with, or has received honoraria or travel or accommodation expenses from Abbvie, Medac, Novocure, Roche, and UCB. MWR has received research funding from UCB. All other authors declare that they have no competing interests.

\section{Additional information}

Supplementary Information The online version contains supplementary material available at https://doi.org/ 10.1038/s41598-021-93663-1.

Correspondence and requests for materials should be addressed to M.W.R.

Reprints and permissions information is available at www.nature.com/reprints.

Publisher's note Springer Nature remains neutral with regard to jurisdictional claims in published maps and institutional affiliations.

(c) (i) Open Access This article is licensed under a Creative Commons Attribution 4.0 International License, which permits use, sharing, adaptation, distribution and reproduction in any medium or format, as long as you give appropriate credit to the original author(s) and the source, provide a link to the Creative Commons licence, and indicate if changes were made. The images or other third party material in this article are included in the article's Creative Commons licence, unless indicated otherwise in a credit line to the material. If material is not included in the article's Creative Commons licence and your intended use is not permitted by statutory regulation or exceeds the permitted use, you will need to obtain permission directly from the copyright holder. To view a copy of this licence, visit http://creativecommons.org/licenses/by/4.0/.

(C) The Author(s) 2021 